\title{
A Sharp RIP Condition for Orthogonal Matching Pursuit
}

\author{
Wei Dan \\ School of Mathematics and Statistics, Guangdong University of Finance \& Economics, Guangzhou 510320, China \\ Correspondence should be addressed to Wei Dan; wdan@gdcc.edu.cn
}

Received 3 July 2013; Revised 14 September 2013; Accepted 5 October 2013

Academic Editor: Sergei V. Pereverzyev

Copyright (C) 2013 Wei Dan. This is an open access article distributed under the Creative Commons Attribution License, which permits unrestricted use, distribution, and reproduction in any medium, provided the original work is properly cited.

A restricted isometry property (RIP) condition $\delta_{K}+\sqrt{K} \theta_{K, 1}<1$ is known to be sufficient for orthogonal matching pursuit (OMP) to exactly recover every $K$-sparse signal $x$ from measurements $y=\Phi x$. This paper is devoted to demonstrate that this condition is sharp. We construct a specific matrix with $\delta_{K}+\sqrt{K} \theta_{K, 1}=1$ such that OMP cannot exactly recover some $K$-sparse signals.

\section{Introduction}

Compressive sampling (compressed sensing, CS) is known as a new type of sampling theory that one can reconstruct a high dimensional spare signal from a small number of linear measurements at the sub-Nyquist rate [1-3]. Nowadays, the CS technique has attracted considerable attention from across a wide array of fields like applied mathematics, statistics, and engineering, including signal processing areas such as MR imaging, speech processing, and analog to digital conversion. The basic problem in CS is to reconstruct the unknown sparse signal $x$ from measurements:

$$
y=\Phi x
$$

where $\Phi$ is an $M \times N(M \ll N)$ sampling matrix. Suppose $\Phi=\left(\Phi_{1}, \Phi_{2}, \ldots, \Phi_{N}\right)$, where $\Phi_{i}$ denotes the $i$ th column of $\Phi$. Throughout the paper, we will assume that the columns of $\Phi$ are normalized; that is, $\left\|\Phi_{i}\right\|_{2}=1$ for $i=1,2, \ldots, N$.

It is well understood that under some assumptions on the sampling matrix $\Phi$, the unknown sparse signal $x$ can be reconstructed by solving the $l_{0}$-minimization problem:

$$
\min \|x\|_{0} \quad \text { subject to } y=\Phi x \text {, }
$$

where $\|x\|_{0}$ denotes the number of nonzero entries of $x$. We say a signal $x$ is $K$-sparse when $\|x\|_{0} \leq K$.

However, the optimization problem is NP-hard, so one seeks computationally efficient algorithms to approximate the sparse signal $x$, such as greedy algorithm, $l_{1}$ minimization, and $l_{p}(0<p<1)$ minimization [4-6].
Orthogonal matching pursuit (OMP), which is a canonical greedy algorithm, has receive much attention in solving the problem (2), due to its ease of implementation and low complexity. Algorithm 1 can be described below. Until recently, many popular generalizations of OMP are introduced, for example, OMMP and KOMP; for details, see [7, 8].

The mutual incoherence property (MIP) introduced in [9] is an important tool to analyze the performance of OMP. The MIP requires the mutual coherence $\mu$ of the sampling matrix $\Phi$ to be small, where $\mu$ is defined as

$$
\mu=\max _{i \neq j}\left|\Phi_{i}^{T} \Phi_{j}\right|
$$

Tropp has shown that the MIP condition $(2 K-1) \mu<1$ is sufficient for OMP to exactly recover every $K$-sparse signal [6]. This condition is proved to be sharp in [10].

The restricted isometry property (RIP) is also widely used in studying a large number of algorithms for sparse recovery in CS, which is introduced in [11]. A matrix $\Phi$ satisfies the RIP of order $K$ with the restricted isometry constant (RIC) $\delta_{K}$ if $\delta_{K}$ is the smallest constant such that

$$
\left(1-\delta_{K}\right)\|x\|_{2}^{2} \leq\|\Phi x\|_{2}^{2} \leq\left(1+\delta_{K}\right)\|x\|_{2}^{2}
$$

holds for all $K$-sparse signal $x$. A related quantity of the restricted orthogonality constant (ROC) $\theta_{K, K^{\prime}}$ is defined as the smallest quantity such that

$$
\left|\left\langle\Phi x, \Phi x^{\prime}\right\rangle\right| \leq \theta_{K, K^{\prime}}\|x\|_{2} \cdot\left\|x^{\prime}\right\|_{2}
$$


Input: Sampling matrix $\Phi$, observation $y$

Output: Reconstructed sparse vector $x^{*}$ and index set

INITIALIZATION: Let the index set $\Omega_{0}=\varnothing$ and the residual $r_{0}=y$. Let the iteration counter $t=1$.

IDENTIFICATION: Choose the index $i$ subject to $\left|\Phi_{i}^{T} r_{t-1}\right|>\max _{j \neq i}\left|\Phi_{j}^{T} r_{t-1}\right|$.

UPDATE: Add the new index $i$ to the index set: $\Omega_{t}=\Omega_{t-1} \cup i$, and update the signal and the residual $\left.x_{t}\right|_{\Omega_{t}}=\arg \min _{z}|| y-\Phi_{\Omega_{t}} z \|_{2},\left.\quad x_{t}\right|_{\Omega_{t}}=0 ;$

$r_{t}=y-\Phi x_{t}$.

If $r_{t}=0$, stop the algorithm. Otherwise, update the iteration counter $t=t+1$ and return to Step IDENTIFICATION.

Algorithm 1: Orthogonal matching pursuit-OMP $(\Phi, y)$.

holds for all disjoint support $K$-sparse signal $x$ and $K^{\prime}$-sparse signal $x^{\prime}$. It is first shown by Davenport and Wakin that the RIP condition

$$
\delta_{K+1}<\frac{1}{3 \sqrt{K}}
$$

can guarantee that OMP will exactly recover every $K$-sparse signal [12]. The sufficient condition is then improved to $\delta_{K+1}<1 /(1+2 \sqrt{K})[13], \delta_{K+1}<1 / \sqrt{2 K}[14], \delta_{K+1}<$ $1 /(1+\sqrt{K})$ [7], and $\delta_{K}+\sqrt{K} \theta_{K, 1}<1[15,16]$. By contrast, Mo and Shen have given a counterexample, a matrix with $\delta_{K+1}=1 / \sqrt{K}$ where OMP fails for some $K$-sparse signals [17]. The main result of this note is to show that the sufficient RIP condition

$$
\delta_{K}+\sqrt{K} \theta_{K, 1}<1
$$

is sharp for OMP.

\section{Main Result}

Theorem 1. For any given positive integer $K \geq 1$, there exist a $K$-sparse signal $x$ and a matrix $\Phi$ with the restricted isometry constant

$$
\delta_{K}+\sqrt{K} \theta_{K, 1}=1
$$

for which OMP fails in $K$ iterations.

Proof. For any given positive integer $K \geq 1$, let

$$
\Phi=\left(\Phi_{i j}\right)_{(2 K-1) \times(2 K-1)}
$$

where

$$
\Phi_{i j}= \begin{cases}0 & (i<j), \\ \sqrt{\frac{2 K}{2 K-1}} \cdot\left(-\frac{i}{\sqrt{i(i+1)}}\right) & (i=j), \\ \sqrt{\frac{2 K}{2 K-1} \cdot \frac{1}{\sqrt{i(i+1)}}} & (i>j) .\end{cases}
$$

By simple calculation, we can get

$$
\begin{aligned}
\left\|\Phi_{j}\right\|_{2}^{2}= & \frac{2 K}{2 K-1} \cdot\left(\frac{j^{2}}{j(j+1)}+\sum_{i=j+1}^{2 K-1} \frac{1}{i(i+1)}\right) \\
= & \frac{2 K}{2 K-1} \\
& \cdot\left(\frac{j}{j+1}+\frac{1}{j+1}-\frac{1}{j+2}\right. \\
= & \left.+\cdots+\frac{1}{2 K-1}-\frac{1}{2 K}\right) \\
&
\end{aligned}
$$

$$
\begin{aligned}
&\left\langle\Phi_{l}, \Phi_{j}\right\rangle= \frac{2 K}{2 K-1} \cdot\left(-\frac{j}{j(j+1)}+\sum_{i=j+1}^{2 K-1} \frac{1}{i(i+1)}\right) \\
&= \frac{2 K}{2 K-1} \\
& \cdot\left(-\frac{1}{j+1}+\frac{1}{j+1}-\frac{1}{j+2}\right. \\
&\left.+\cdots+\frac{1}{2 K-1}-\frac{1}{2 K}\right) \\
&=-\frac{1}{2 K-1}
\end{aligned}
$$

for any integers $1 \leq l<j \leq 2 K-1$.

Thus, for any index set $\Lambda$ whose cardinality is $K$, we have

$$
\Phi_{\Lambda}^{T} \Phi_{\Lambda}=\left(\begin{array}{cccc}
1 & -\frac{1}{2 K-1} & \cdots & -\frac{1}{2 K-1} \\
-\frac{1}{2 K-1} & \cdots & -\frac{1}{2 K-1} & 1
\end{array}\right) .
$$

It is obvious that the eigenvalues $\left\{\lambda_{i}\right\}_{i=1}^{K}$ of $\Phi_{\Lambda}^{T} \Phi_{\Lambda}$ are

$$
\begin{gathered}
\lambda_{1}=\cdots=\lambda_{K-1}=1+\frac{1}{2 K-1}, \\
\lambda_{K}=1-\frac{K-1}{2 K-1} .
\end{gathered}
$$


Therefore, the restricted isometry constant $\delta_{K}$ of $\Phi$ is $(K-$ $1) /(2 K-1)$.

Now, we turn to calculate the restricted orthogonality constant $\theta_{K, 1}$. In view of (11), we may, without loss of generality, assume that $x=\left(x_{1}, \ldots, x_{K}, 0, \ldots, 0\right)^{T}$ and $x^{\prime}=$ $\left(0, \ldots, 0, x_{K+1}^{\prime} 0, \ldots, 0\right)^{T}$. We have

$$
\begin{aligned}
\theta_{K, 1} & =\max \frac{\left|\left\langle\Phi x, \Phi x^{\prime}\right\rangle\right|}{\|x\|_{2} \cdot\left\|x^{\prime}\right\|_{2}} \\
& =\max \frac{\left|\left\langle\Phi x, \Phi_{K+1}\right\rangle\right|}{\|x\|_{2}} \\
& =\max \frac{1}{2 K-1} \cdot \frac{\left|\sum_{i=1}^{K} x_{i}\right|}{\|x\|_{2}} \\
& =\frac{\sqrt{K}}{2 K-1} .
\end{aligned}
$$

The last equality holds when $x_{1}=\cdots=x_{K}$. It is easy to check that

$$
\begin{aligned}
& \delta_{K}+\sqrt{K} \theta_{K, 1}=\frac{K-1}{2 K-1}+\sqrt{K} \cdot \frac{\sqrt{K}}{2 K-1}=1 \\
& \text { Let } x=\underbrace{(1, \ldots, 1}_{K}, 0, \ldots, 0)^{T} \in \mathbf{R}^{2 K-1} \text {; we have } \\
& \left|S_{j}\right|=\left|\left\langle\Phi x, \Phi_{j}\right\rangle\right|=\frac{K}{2 K-1}, \quad \forall j \in\{1,2, \ldots, 2 K-1\} .
\end{aligned}
$$

This implies that OMP fails in the first iteration. The proof is complete.

\section{Discussion}

In this paper, we showed that the RIP condition $\delta_{K}+$ $\sqrt{K} \theta_{K, 1}<1$ is sharp for orthogonal matching pursuit to exactly recover every $K$-sparse signal $x$ from measurements $y=\Phi x$. It is worth discussing the relations between our sharp RIP condition and that in two relative papers [10, 17]. First of all, it follows from the facts that $\delta_{K}<(K-1) \mu$ and $\theta_{K, 1}<\sqrt{K} \mu$ that the sharp RIP condition $\delta_{K}+\sqrt{K} \theta_{K, 1}<1$ in this paper is weaker than the sharp MIP condition $(2 K-$ $1) \mu<1$ in [10]. Moreover, our result is also stronger than the previous RIP condition. The condition $\delta_{K}+\sqrt{K} \theta_{K, 1}<1$ in this paper is necessary and sufficient for OMP, while the previous necessary RIP condition $\delta_{K}<1 / \sqrt{K}$ in [17] is not sufficient. Therefore, the result in the paper may guide the practitioners to apply OMP properly in sparse recovery.

\section{Acknowledgments}

This work was supported by the National Natural Science Foundation of China (Grant nos. 11271060, U0935004, U1135003, 11071031, and 11290143, 11101096), the Guangdong Natural Science Foundation (Grant no. S2012010010376), and the Guangdong University and Colleges Technology Innovation Projects (Grant no. 2012KJCX0048).

\section{References}

[1] E. J. Candès, J. Romberg, and T. Tao, "Robust uncertainty principles: exact signal reconstruction from highly incomplete frequency information," IEEE Transactions on Information Theory, vol. 52, no. 2, pp. 489-509, 2006.

[2] D. L. Donoho, "Compressed sensing," IEEE Transactions on Information Theory, vol. 52, no. 4, pp. 1289-1306, 2006.

[3] Z. Xu, "Compressed sensing: a survey," Scientia Sinica Mathematica, vol. 42, no. 9, pp. 865-877, 2012.

[4] D. L. Donoho and M. Elad, "Optimally sparse representation in general (nonorthogonal) dictionaries via $l_{1}$ minimization," Proceedings of the National Academy of Sciences of the United States of America, vol. 100, no. 5, pp. 2197-2202, 2003.

[5] R. Saab and Y. Yılmaz, "Sparse recovery by non-convex optimization-instance optimality," Applied and Computational Harmonic Analysis, vol. 29, no. 1, pp. 30-48, 2010.

[6] J. A. Tropp, "Greed is good: algorithmic results for sparse approximation," IEEE Transactions on Information Theory, vol. 50, no. 10, pp. 2231-2242, 2004.

[7] R. Maleh, "Improved RIP analysis of orthogonal matching pursuit," 2011, http://arxiv.org/abs/1102.4311.

[8] Z. Xu, "The performance of orthogonal multi-matching pursuit under RIP," 2012, http://arxiv.org/abs/1210.5323.

[9] D. L. Donoho and X. Huo, "Uncertainty principles and ideal atomic decomposition," IEEE Transactions on Information Theory, vol. 47, no. 7, pp. 2845-2862, 2001.

[10] T. T. Cai, L. Wang, and G. Xu, "Stable recovery of sparse signals and an oracle inequality," IEEE Transactions on Information Theory, vol. 56, no. 7, pp. 3516-3522, 2010.

[11] E. J. Candes and T. Tao, "Decoding by linear programming," IEEE Transactions on Information Theory, vol. 51, no. 12, pp. 4203-4215, 2005.

[12] M. A. Davenport and M. B. Wakin, "Analysis of orthogonal matching pursuit using the restricted isometry property," IEEE Transactions on Information Theory, vol. 56, no. 9, pp. 43954401, 2010.

[13] S. Huang and J. Zhu, "Recovery of sparse signals using OMP and its variants: convergence analysis based on RIP," Inverse Problems, vol. 27, no. 3, Article ID 035003, 14 pages, 2011.

[14] E. Liu and V. N. Temlyakov, "The orthogonal super greedy algorithm and applications in compressed sensing," IEEE Transactions on Information Theory, vol. 58, no. 4, pp. 2040-2047, 2012.

[15] W. Dan and R. H. Wang, "Robustness of orthogonal matching pursuit under restricted isometry property," Science China Mathematics, vol. 56, 2013.

[16] J. Wang and B. Shim, "Improved recovery bounds of orthogonal matching pursuit using restricted isometry property," 2012, http://arxiv-web3.library.cornell.edu/abs/1211.4293vl.

[17] Q. Mo and Y. Shen, "A remark on the restricted isometry property in orthogonal matching pursuit," IEEE Transactions on Information Theory, vol. 58, no. 6, pp. 3654-3656, 2012. 


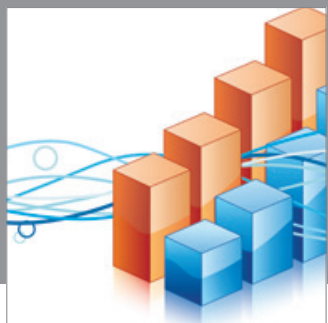

Advances in

Operations Research

mansans

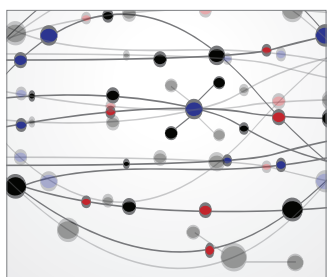

The Scientific World Journal
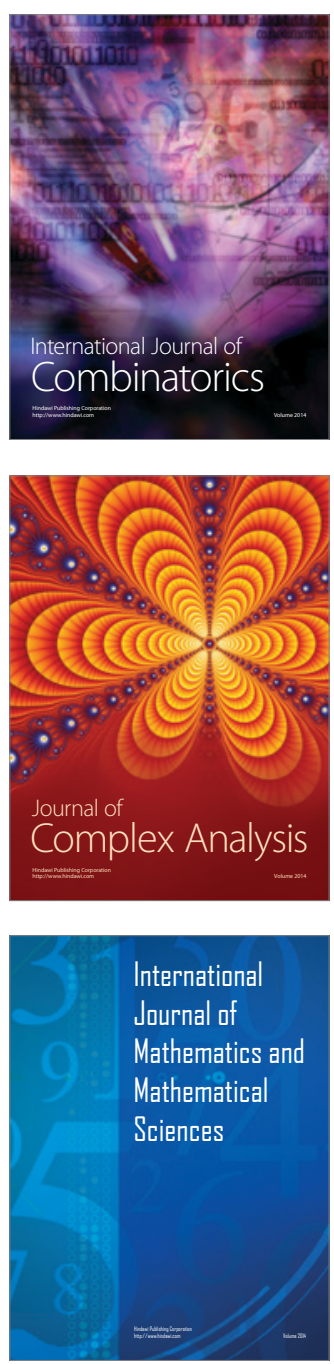
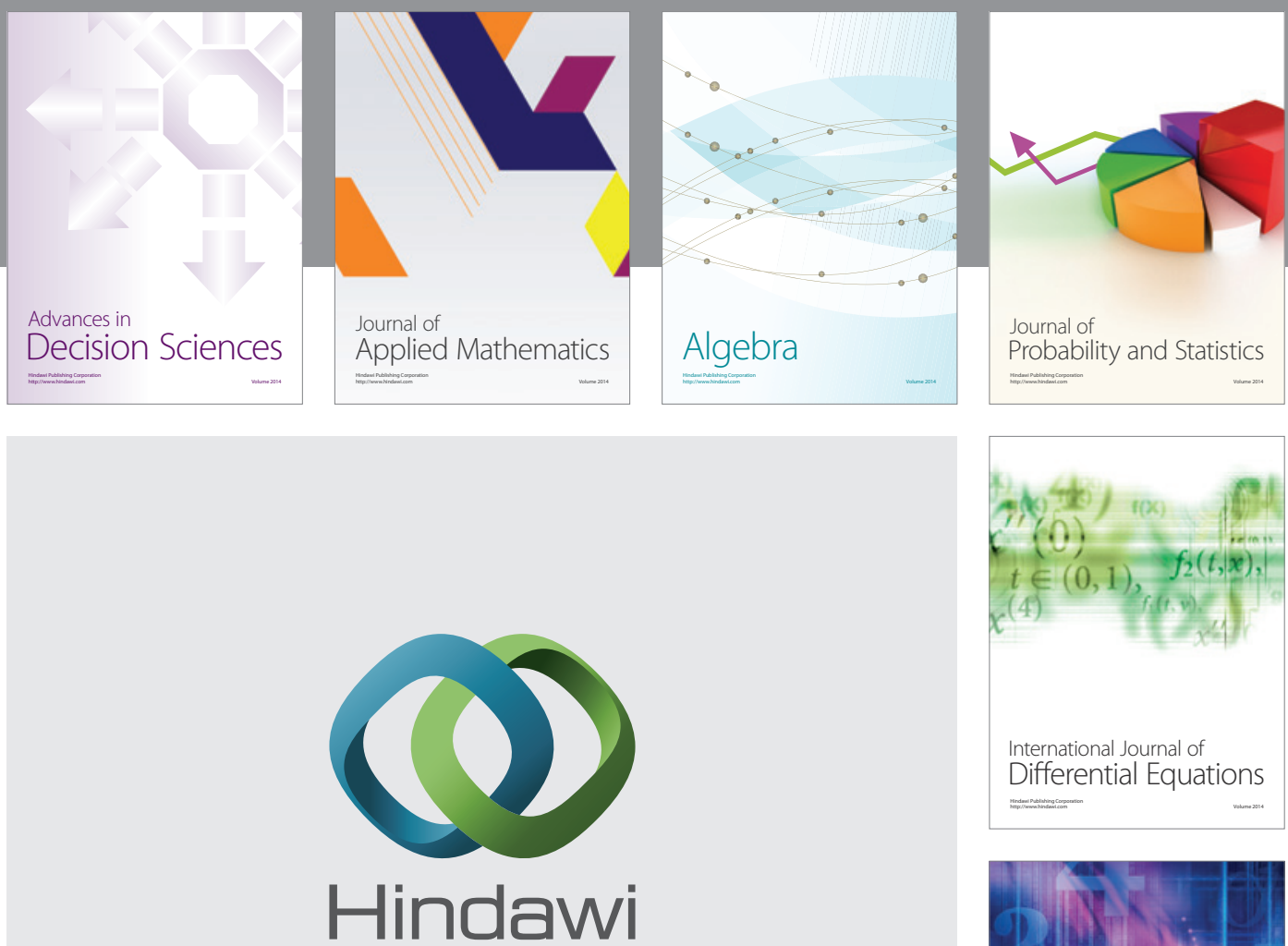

Submit your manuscripts at http://www.hindawi.com
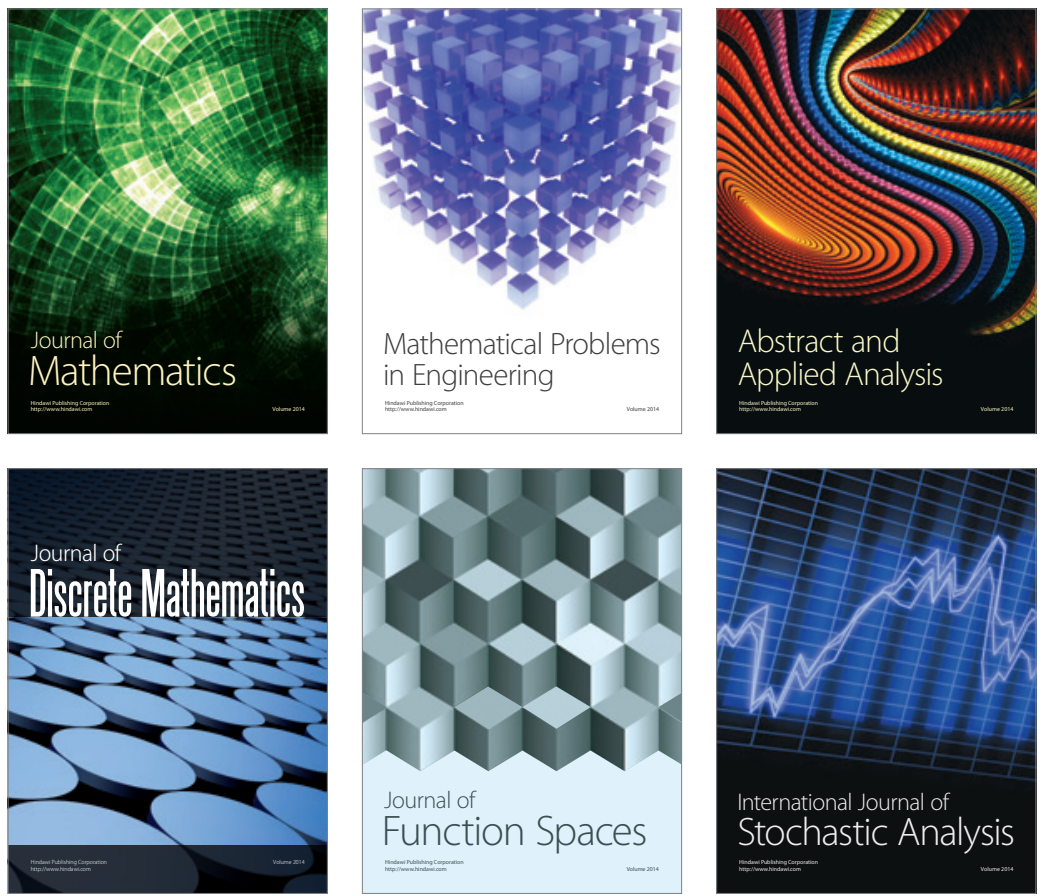

Journal of

Function Spaces

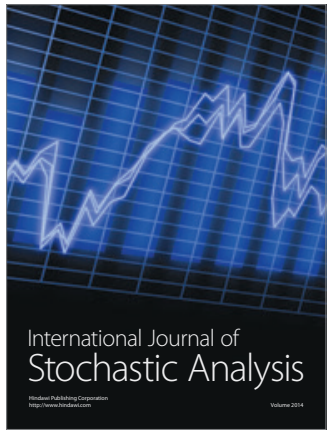

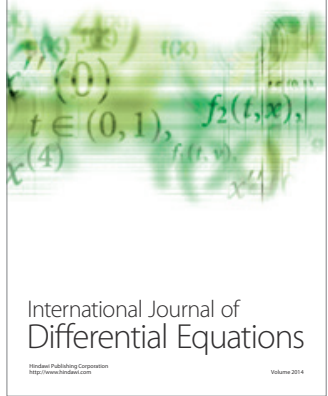
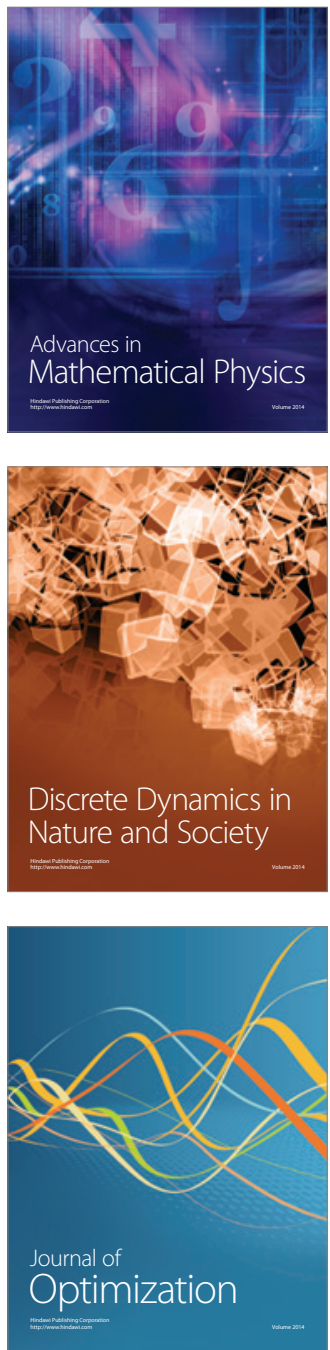